\title{
Application Virtualization for Teaching Hospitals in Nigeria
}

Desmennu Senanu Rita

Dept. of Computer and Info. Sci.

\author{
Covenant University \\ Ota, Ogun State \\ Nigeria \\ $+234-8067601114$ \\ senaritad@gmail.com
}

\author{
Ikhu-Omoregbe Nicholas \\ Dept. of Computer and Info. Sci \\ Covenant University \\ Ota, Ogun State \\ Nigeria \\ +234-8060093448 \\ nomoregbe@gmail.com
}

\begin{abstract}
Information technology has improved operations management globally. The health sector has benefited from this revolution through the introduction of eHealth solutions. The cost-effective utilization of Information Technology and flexibility in adapting and adopting organizational changes has posed some challenges to health institutions in developing countries. In the case of Nigeria, the implementation of the National Health Policy entails the delivery of a full-packaged health care system; this package includes health education, maternal, newborn and child healthcare, nutrition and immunization. All these, require record keeping and data storage. The management of massive data storage and its availability on-demand has been sources of concern to health institutions in the country. This has brought about a slow rate in hospital-tohospital collaboration, insecure information exchange between and across institutions and lack of proper accountability in the health sector amongst other challenges.

In this paper we propose a Cloud computing infrastructure which will adopt application virtualization to address the challenges in health care delivery in the country. This is an emerging technology that will provide eHealth solutions as services to tenants; a process known as Software-as-aService (SaaS). The infrastructure should deliver a single application through the browser to thousands of clients or stakeholders using scalable multitenant architecture. This will help to minimize cost, manage healthcare resources effectively, and help with the realization of the Millennium Development Goals (MDGs) on healthcare.
\end{abstract}

\section{Keywords}

Virtualization, SaaS, eHealth, multitenant, Information Technology, Nigeria

\section{INTRODUCTION}

Virtualization is a technology that abstracts away the details of the physical hardware and provides virtualized resources for high-level applications [6]. It is a technique for hiding the physical characteristics of computing resources to simplify the way in which other systems, applications, or end users interact with those resources.

Virtualization is paradigm that implies the abstraction of computing resources. It broadly describes the separation of a resource or request for a service from the underlying physical delivery of that service. It is an innovation that allows the deployment of creative solutions to organizational and business challenges. The application of virtualization in computing has led to the emergence of platform virtualization, virtual memory, application virtualization, network virtualization, storage virtualization and many others [Vmware whitepaper]. A kind of computing that adopts this innovation is Distributed Computing. Distributed computing entails accessing contents and resources over a network without reference to the infrastructure hosting such contents. These contents are managed by content providers. They reside in well managed, monitored and maintained data centers across the globe. Several Distributed computing technologies with resultant applications have evolved over time.

SOA generally refers to design and not implementation. SOA is an architectural style of designing and building reliable distributed systems that deliver functionality as services, with the additional emphasis on loose coupling between interacting services [7]. A service is a discoverable resource that executes a repeatable task, and is described by an externalized service specification [8].

Web services are distributed software components that provide information to applications rather than to humans, through an application-oriented interface [7]. Web services are an implementation technology; they publish details of their functions and interfaces, but keep implementation details private, a feature that makes them applicable to a distributed heterogeneous environment. Web services address discovery and invocation of persistent services; these are services that outlive their clients [9]. They are available until the server is shut down. Cluster computing. Health clusters are locally resident in hospitals. Hospitals 
have made use of inter-connected computers to help facilitate interaction among health personnel. Computing in clusters did not provide efficient use of idle resources, and are highly susceptible to failure as a result of homogenous nodes (single point of failure).

Another technology infrastructure proposed was the Grid approach which provides large-capacity computational power. Healthcare applications on the grid (HealthGrids) include MediGRID [cited in Viezens etal, 2008] and GridSphere portal [cited in Viezens etal, 2008]. NeuGrid which aims to supply neuroscientists with the most advanced ICT tools to help overcome neuro-degenerative diseases such as Alzheimer's, gLite which optimizes the use of heterogeneous computing resources to process medical imaging and RadioTherapyGrid which has been used to optimize the process of cancer treatment are also instances of grid applications in healthcare.

A more recent technology is Cloud computing. A definition of Cloud computing as proposed in [Buyya etal, 2008] denotes a Cloud as a type of parallel and distributed system. It consists of a collection of inter-connected and virtualized computers. It also applies the principle of server virtualization. These computers are dynamically provisioned and presented as one or more unified computing resource(s) based on service-level agreements. These service level agreements are established through negotiation between the service provider and consumers. Cloud computing delivers infrastructure, platform and application as services [Calheiros etal, 2009]. The supercomputing power of a cloud is made available by a network of data centers in which several servers are installed. Cloud computing represents the infrastructure as a "Cloud". Users (tenants and clients) are able to access cloud applications from anywhere in the world on demand (Ondemand computing). This is gradually and rapidly transforming the computing world towards developing software to be consumed as a service (Software-as-a Service: SaaS), rather than to run on their individual computers. There are sets of parameters and features that clearly distinguish Cloud computing [Buyya etal, 2008]. They include:

- $\quad$ Single ownership,

- It is populated by commodity computers and highend severs whose network speed is high-end with low latency and high bandwidth.

- The resources in a Cloud are either centralized or distributed with their capacities provisioned on demand.

- $\quad$ Clouds have strong support for virtualization with high potential for dynamically creating valueadded services with web-service interface and for fail over and content replication.
- Clouds guarantee high-level security and privacy as each application has its own virtual machine and support access control list.

Emerging cloud platforms and technologies include [Buyya etal, 2008]: Amazon Elastic Compute Cloud EC2, Google App Engine, Microsoft Azure, Sun Grid, Aneka, Virtual Workspaces, OpenNebula, CloudSim and Reservoir. cloud computing has its application in social networking, web hosting, gaming portals, business applications, scientific workflows, healthcare, content delivery and data processing and storage.

\subsection{Application Virtualization}

Application virtualization is an all encompassing term that describes software technologies that improve portability, manageability and compatibility of applications by separating them from the underlying platforms on which they are executed. It is a technology that enhances portability, manageability, compatibility and stability of applications. A fully virtualized application is not installed in the traditional sense, although it is still executed as if it is. It transforms applications into a pool of centrallymanaged virtual services that are compatible with other applications without being installed. Physically, an operating system allocates a range of resources and services (e.g memory allocation, drivers,) to applications running on it. Incompatibilities between an application and its operating system can be addressed by either server virtualization or presentation virtualization; but application virtualization addresses incompatibilities between two applications installed on the same instance of an operating system. Application virtualization differs from platform virtualization in that only specific applications are virtualized while the whole operating system is virtualized in platform virtualization. A cloud service can be infrastructure for hosting applications or data storage, a development platform, or even an application that you can get on-demand, either off-site at a provider or built onsite within IT.

Application virtualization improves end user productivity by eliminating or reducing to the barest minimum all of the complexities and support issues associated with delivering and accessing traditional applications. It eliminates application installation, reduces end-user impacts associated with software upgrades, patching and terminations, and helps in application streaming.

\section{2. eHEALTH IN NIGERIA}

\subsection{Nigeria's Health System}

Primary healthcare delivery is the mode of operation of the healthcare system in Nigeria. This also forms an integral part of the National Health Policy. This policy entails the delivery of a full-packaged health care system. This 
package includes health education, maternal, newborn and child healthcare, nutrition and immunization. Effective management of healthcare at the grassroots requires record keeping and data storage. Massive Data storage and availability of such data on-demand have been sources of concern to health institutions. Lack of storage facilities and media have brought about a slow rate in hospital-tohospital collaboration. Also, secure information exchange between and across institutions poses as a great challenge to medical practitioners. This does not enhance collaboration (also referrals) among such institutions. These challenges have brought about a lack of proper accountability in the health sector. A health sector reform was introduced in 2004 to address this issue [Labiran etal, 2008]. As a result, there is an appreciable increase in the resources committed to the health sector. This is to ensure the attainment of the Millennium Development Goals (MDGs) on healthcare.

With the advent and significant advances of Information Technology over time, there have been increasing areas of application. The healthcare sector has had its own share. The emerging field is E-Health. E-Health is an intersection of medical informatics, public health and business. It refers to health services and information delivered or enhanced through the Internet and related technologies.

Nigeria is lagging behind in the adoption of this emerging field of technology. This is as a result of the high costs of acquiring such solutions. Such costs include application cost, hardware cost, labor cost, maintenance cost. This has made health institutions and health professionals comfortable with the traditional ways of carrying out their routines.

\subsection{Existing eHealth Solutions in Nigeria}

In Nigeria, a popular solution that has been developed and deployed across hospitals is MINPHIS. MINPHIS is an acronym for Made-In-Nigeria Primary healthcare and Hospital Information System [1][2][3]. It was developed in 1989 as a product of collaboration between Nigeria (Obafemi Awolowo University, Ile-Ife [4]) and Finland research and development project on health informatics. Its second version is currently available. It has been deployed across eight (8) Nigerian teaching hospitals as of July 2005, and it's suitable for the different types of health facilities as a result of its scalability.

In 2009, the pilot scheme of the e-health project was launched in Lagos state [5]. The Lagos State Health Management Information System (HMIS) project is a broad-based modular Hospital Administration System Database solution that allows the operational procedures and stream of patients in a health facility to be coordinated thus ensuring prompt and effective healthcare delivery. The project is currently in its second phase, and its connecting more hospitals. The links to the diagnostic modules ensures doctors online can order tests while results can be returned via the same channel. Critical minutes saved by this prompt information availability will save a lot of lives as will be shown in statistical data to be maintained.

\section{METHODOLOGY}

\subsection{Cloud Architecture}

Cloud computing architecture is generally made up of three layers. Each layer is loosely coupled with the layers above and below. This makes the architecture more modular, and allows each layer to operate separately. The architecture can be compared with the OSI model for network protocols. The layers are discussed as follows and depicted in Figure 1.

- Application layer: this represents the customer's actual interface. It delivers applications via the virtualized platform and infrastructure layers. The services in this layer are of two types; software-asa service and on-demand web services.

- Platform layer: it resides on top of the Infrastructure layer and provides value-added services from both technical and business perspective. It consists of operating systems and application frameworks. This layer minimizes the burden of deploying applications directly into VM containers. Platform services are of two types: Development platforms and Business platforms.

- Infrastructure layer: this is an important layer of the architecture. Virtualization takes place at this layer. It provides the enabling technologies for the cloud. It provisions a pool of storage and computing resources through virtualization technology. In this layer also reside the physical resources (hardware) of the cloud. These include the physical servers, routers, switches, power and cooling systems.

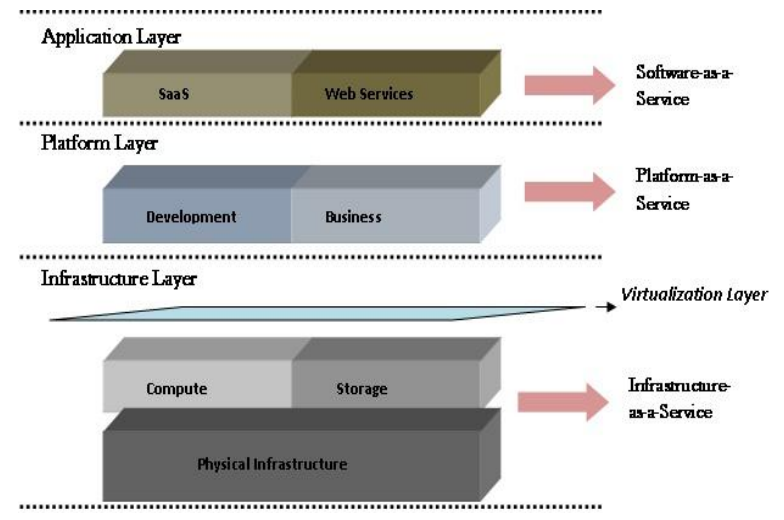

Figure 1: Cloud Architecture 


\subsection{Cloud Computing Approach}

A cloud service can be infrastructure for hosting applications or data storage, a development platform, or even an application that you can get on-demand, either offsite at a provider, or built onsite within IT. The methodology adopted in this design is Software-as-Service (SaaS) or Application Virtualization. It employs virtualization alongside multitenancy. This methodology delivers a single application through the browser to thousands of customers (users) using a scalable multitenant architecture. SaaS architectures are generally classified to one of four "maturity levels", where each level differs from the previous by unique attributes as represented below.

Maturity Level $4 \longrightarrow$\begin{tabular}{l|} 
Scalable, Configurable, \\
Multitenant Efficient
\end{tabular}

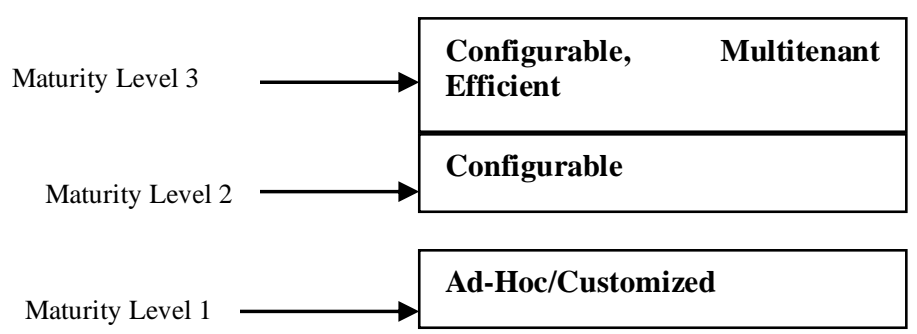

Figure 2: SaaS Architecture

This works adopts SaaS' maturity level 4. Multitenant architecture allows several customers (health institutions) to share infrastructure, without the customers being aware of it. Scalability allows increase or reduction in the system's capacity to match demand per time, without the need for any further alteration of applications. This can be done without compromising the privacy and security of each institution's data. This methodology ensures no upfront investment in servers or software licensing for clients or users; while with just one application to maintain, providers enjoy minimum costs compared to conventional hosting.

\subsection{Proposed Health-Cloud Architecture}

The proposed Health Cloud runs on a multitenant architecture. This software architecture is one where a single instance of the software runs on a SaaS vendor's server, serving multiple client organizations (tenants) as shown in Figure 2. The multitenant data will be managed using a single database. The database server will be virtualized, thereby provisioning virtual servers for each tenant, these resources will be provided as services. The teaching hospitals in Nigeria are the tenants of the cloud. Encryption and permission are the security patterns that will be applied to Health-Cloud. A database access account will be created for each health institution, and AccessControl Lists (ACL) will be used to grant each of these institution accounts access to the database objects they are allowed to use. Within the health application itself, rolebased access is put in place to prevent unauthorized access to data. Health Institutions can connect from any geographic location to the cloud and utilize needed resources.

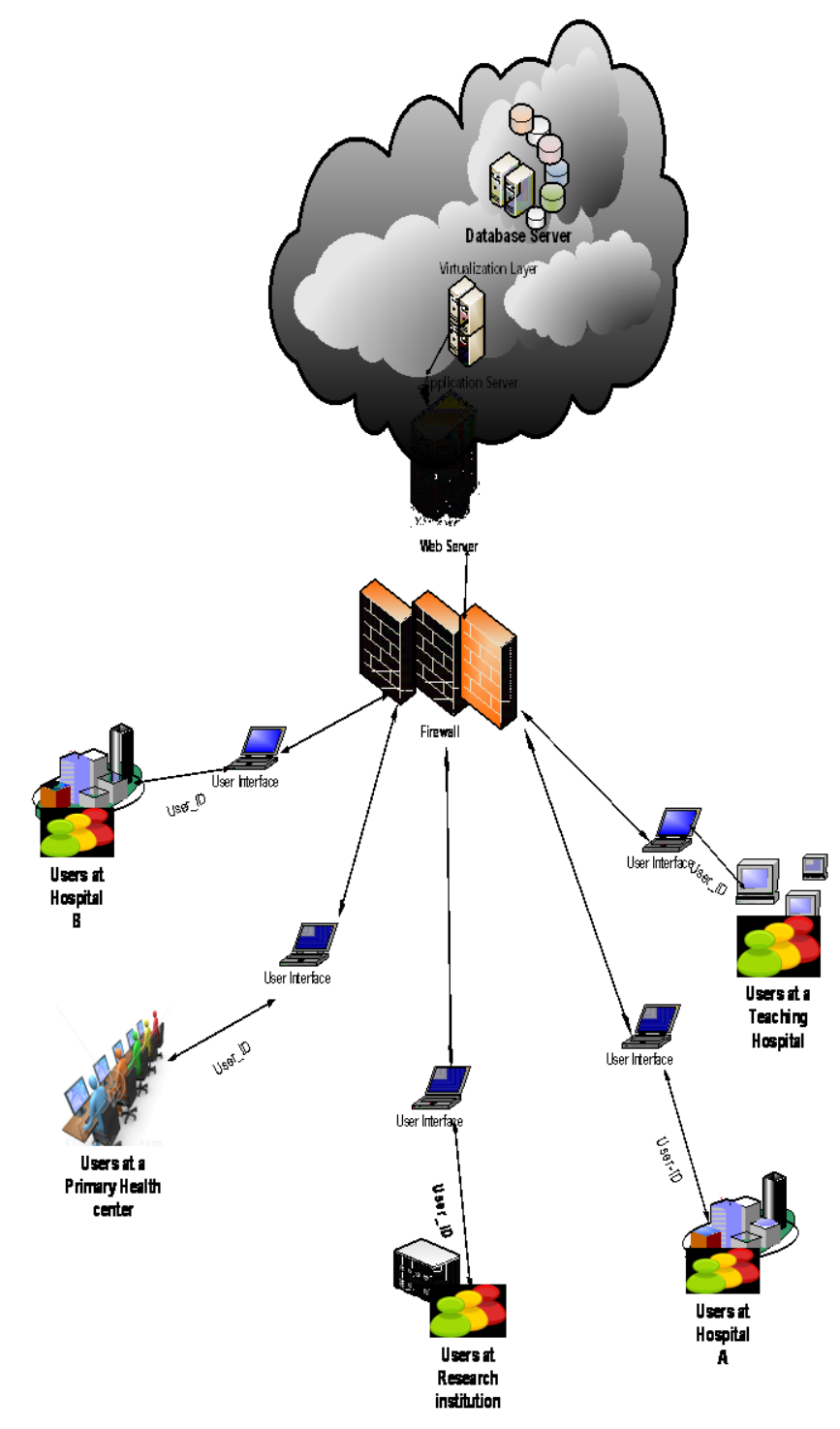

Figure 3: Proposed Health-Cloud framework

\subsection{Relevance of Health application Virtualization}

eHealth application virtualization will help to streamline the deployment process, making it faster and easier. It will also create application-specific copies of all shared resources that are isolated into their own virtual environments while allowing interaction with local system resources but prevents applications from overwriting the resources of other applications. Generally, it will provide the following benefits: application isolation, improved 
provisioning, improved auditing, improved IT control, and improved end user agility. Hosting application virtualization in the cloud will also reduce cost for health institutions as each customer pays for what he uses, thus facilitating and accelerating the adoption and adaptation rate of eHealth solutions.

\section{CONCLUSION}

The introduction and adoption of the Health-Cloud will ensure all individuals have equal rights to primary healthcare so no one is underinsured or over insured. In the long run, it will help to meet the Millennium Development Goals (MDGs) on healthcare delivery for all.

\section{REFERENCES}

[1] Buyya R., Yeo C.S., Venugopal S., Broberg J., and Brandic I. (2008), "Cloud Computing and Emerging IT Platforms:Vision, Hype, and Reality for Delivering Computing as the 5th Utility", In Proceedings of the $10^{\text {th }}$ IEEE International Conference on High Performance Computing and Communications, 2008. http://www.gridbus.org/reports/CloudITPlatforms2008 .pdf.

[2] Calheiros R.N., Ranjan R., De Rose C.A.F., Buyya R.(2009), "CloudSim: A Novel Framework for Modelling and Simulation of Cloud Computing Infrastructures and Services".

[3] Labiran A., Mafe M., Onajole B., Lambo E. (2008), "Health Workforce Country Profile for Nigeria", First Edition, Nigeria.

[4] Viezens F. and Sax U., (2008), "Collaboration at the Transition of Personalized Medicine, Electronic Health Record and HealthGRIDS- GRID Services and Applications for the Future", In Blobel B. etal (ed.), "ehealth: Combining Health Telematics, Telemedicine, Biomedical Engineering and Bioinformatics to the Edge, CeHR Conference Proceedings 2007”, Berlin: Akademische Verlagsgesellschaft Aka GmbH, 350 pages.

[5] vmWare White paper, "Virtualization Overview", http://www.nitro.ca/images/pdf/virtualization.pdf.

[6] Qi Zhang etal (2010), "Cloud Computing: state-of-the-art and research challenges", J. of Internet Services and Application, 1: 7-18

[7] Latha Srinivasan, Jem Treadwell, "An Overview of ServiceOriented Architecture, Web Services and Grid Computing", HP Software Global Business Unit, $3^{\text {rd }}$ November, 2005.

[8] Bertrand Portier, "SOA Terminology overview, Part 1: Service, Architecture, Governance and Business terms", 24 ${ }^{\text {th }}$ May, 2007.

http://www.ibm.com/developerworks/webservices/libr ary/ws-soa-term1/
[9] http://www.cnaf.infn.it/ ferrari/seminari/griglie04/lezi one02-ogsa.pdf

Web References

1. http://minphis.4t.com/ (accessed $16^{\text {th }}$ August, 2009)

2. http://www.egov4dev.org/minphis.htm (accessed $16^{\text {th }}$ August, 2009)

3. http://www.uku.fi/tike/indehela/Nig2004report.ht m (accessed $16^{\text {th }}$ August, 2009)

4. http://www.oauife.edu.ng/ (accessed $16^{\text {th }}$ August, 2009)

5. http://pmnewsnigeria.com/2010/08/13/lagosbegins-e-health-project/

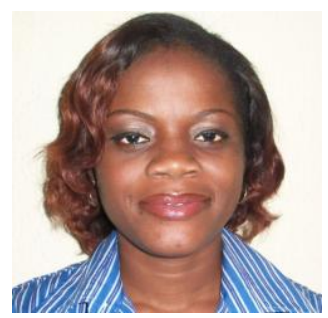

Desmennu Senanu Rita holds a M.Sc in Management Information Systems (MIS). Her research areas include Requirements

Engineering with special focus on Data modeling and System Analysis and Design. She is currently pursuing a Ph.D in the area of e-Health in Covenant University, Ota, Ogun state, Nigeria.

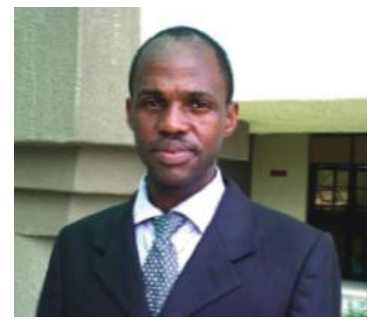

Ikhu-Omoregbe, Nicholas holds a B.Sc degree in Computer Science from the University of Benin, Benin City, an M.Sc. degree in Computer Sciences from the University of Lagos, and a PhD degree in Computer Science from Covenant University, Ota, Nigeria. His research interests include: Software Engineering, Mobile Computing, Multimedia technologies, Mobile Healthcare and Telemedicine Systems, and Soft Computing. He currently lectures in the Department of Computer and Information Systems, Covenant University, Ota, and has taught at Baden-Wurttemberg Cooperative State University, Heidenheim as a visiting lecturer in the area of e-Health Systems. 